\title{
Uso de antagonistas del calcio en el tratamiento del síndrome uretral en las mujeres con urgencia e incontinencia urinaria
}

\author{
Granados Loarca EA.
}

Servicio de Urología. Torre Profesional. Quetzaltenango, Guatemala.

Actas Urol Esp 2006; 30 (4): 406-408

\section{RESUMEN}

\section{USO DE ANTAGONISTAS DEL CALCIO EN EL TRATAMIENTO DEL SÍNDROME URETRALEN LAS MUJERES CON URGENCIA E INCONTINENCIA URINARIA}

Objetivo: Se evalúa la acción de los antagonistas del calcio en el Síndrome Uretral de la mujer (incontinencia, urgencia, disuria).

Pacientes y Metodos: En 60 pacientes de sexo femenino que acudieron con Síndrome Uretral. Se utilizo la Cinarizina en 20 pacientes, y la Flunarizina en 20 pacientes. Y en 20 pacientes se utilizó placebo.

Resultados: En las pacientes que recibieron Flunarizina como Cinarizina se observo una reducción de la incontinencia de orina, de la urgencia, y de la disuria, la micción fue mas prolongada y más fácil, el número de las micciones se redujo al día, comparados con las pacientes tratadas con placebo.

Conclusiones: Los antagonistas del calcio pueden ser útiles en aquellas pacientes con síndrome uretral en especial en las mujeres mayores.

Palabras Clave: Antagonistas Calcio. Sindrome Uretral.

\section{ABSTRACT}

\section{CALCIUM ANTAGONIST IN THE URETHRAL SYNDROME OF THE WOMAN WITH INCONTINENCE AND URGENCE}

Objective: The action of the antagonists of the calcium was evaluated in the Urethral Syndrome of the woman (incontinence, urgency, disury).

Patient and Methods: In 60 female's patients that went for urethral syndrome. I used the Cinarizina in 20 patients, and the Flunarizina in 20 patients. And in 20 patients were used placebo.

Results: In the patients that used Flunarizina and Cinarizina were observed a reduction of the urine incontinence, of the urgency, and the disury, the micturition was lingering and easy, the number of the micturitions decreased by the morning, compared with the patients that used placebo.

Conclusions: The antagonists of the calcium can be useful in those patients with urethral syndrome especially the biggest women.

Keywords: Calcium Antagonists. Urethral Syndrome.

$\mathrm{E}$ sindrome uretral en la mujer puede causar en ocasiones serias molestias en estas pacientes, por lo que se han utilizado múltiples medicamentos para tratar de aliviar en especial la disuria, en esta serie se utilizó los calcio- antagonistas y se presenta sus efectos.

\section{PACIENTES Y MÉTODOS}

En 40 pacientes de sexo femenino que acudieron por Sindrome uretral, urgencia urinaria, disuria, e incontinencia de orina. Se utilizo antagonistas del calcio tales como la Cinarizina a dosis 75 mg/día en 20 pacientes, y la Flunarizina a dosis 
de $10 \mathrm{mg} /$ día en 20 pacientes. $\mathrm{Y}$ en 20 pacientes se utilizo placebo. Todas habían recibido algún tipo de tratamiento medico, dilataciones y/o cirugía, Tabla 1. La edad de las pacientes osciló entre 24-72 años (media 53 años). A 6 pacientes se les había efectuado histerectomía, a 4 se le había efectuado colporrafia anterior. El motivo de consulta se presenta en la Tabla 2. El tiempo de tratamiento oscilo entre 4-24 semanas.

\section{RESULTADOS}

Las pacientes presentaron cambios en el vaciamiento vesical, tanto en las pacientes que recibieron Flunarizina como Cinarizina. Se observó una reducción de la incontinencia de orina, de la urgencia y principalmente de la disuria, la micción fue mas prolongada y más fácil, el número de las micciones diarias se redujeron, comparado con las pacientes tratadas con placebo, esto se constató en base a un cuestionario que se les facilitó a las pacientes, donde tenían que contestar como se sentían con el tratamiento: excelente, bien, regular y mal (Fig. 1). Los efectos colaterales observados en las pacientes tratadas con vasodilatadores (Flunarizina y Cinarizina) fueron somnolencia en 5 pacientes, cefalea en 5 pacientes, dolor abdominal en 2 pacientes, y sequedad de boca en 2 pacientes. La cistometria fue normal en la mayoría de estas pacientes (Tabla 3). El tiempo de tratamiento ideal fue de 4 a 8 semanas. El tiempo de seguimiento fue de 36 meses post-tratamiento, en este tiempo ninguna volvió a presentar síntomas.
Tabla 1

\begin{tabular}{lccc}
\hline $\begin{array}{l}\text { Antecedentes } \\
\text { Tratamiento }\end{array}$ & Flunarizina & Cinarizina & Placebo \\
\hline $\begin{array}{l}\text { Medico } \\
\text { Anticolinergicos }\end{array}$ & 20 & 20 & 19 \\
Alfa-bloqueadores & 12 & 13 & 10 \\
Antisépticos Urinarios & 19 & 20 & 17 \\
Analgésicos y desinflamatorios & 20 & 19 & 19 \\
& & & \\
Dilataciones uretrales & 12 & 10 & 11 \\
Cirugia Incontinencia & & & \\
$\quad$ MMK & 4 & 2 & 1 \\
Burch & 2 & 1 & 1 \\
IVS & 1 & 0 & 1 \\
\hline
\end{tabular}

Tabla 2

\begin{tabular}{lccc}
\hline Motivo de Consulta & Flunarizina & $\begin{array}{c}\text { Cinarizina } \\
\text { No. }\end{array}$ & Placebo \\
\hline Resequedad de Mucosas & 16 & 17 & 17 \\
Incontinencia de orina & 18 & 19 & 19 \\
Urgencia & 20 & 18 & 19 \\
Polaquiuria & 11 & 14 & 11 \\
Disuria & 20 & 19 & 18 \\
ITU & 7 & 6 & 4 \\
Dolor Suprapubico & 18 & 17 & 17 \\
Dolor Lumbar & 9 & 8 & 6 \\
\hline
\end{tabular}

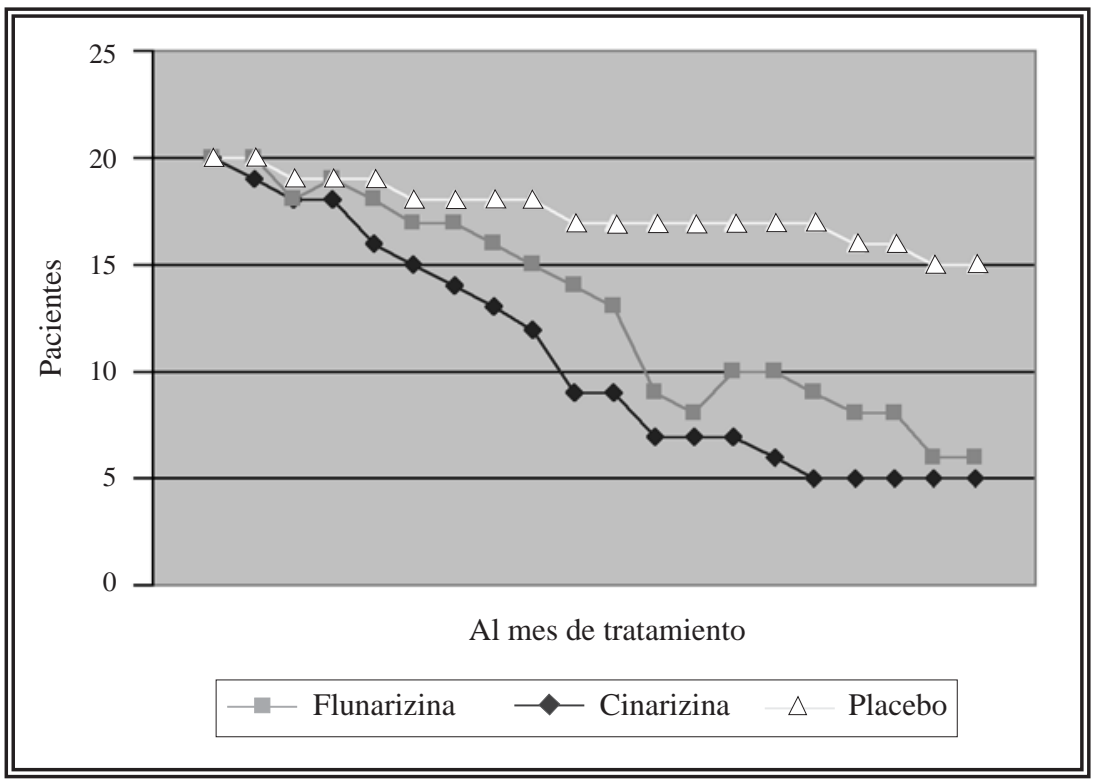

FIGURA 1. Efectos de los antagonistas del calcio sobre el sindrome uretral. 
Tabla 3

\begin{tabular}{lccc}
\hline Cistometrias & Flunarizina & Cinarizina & Placebo \\
\hline Normal & 10 & 11 & 12 \\
$\begin{array}{l}\text { Vejigas de poca } \\
\text { capacidad }\end{array}$ & 2 & 1 & 3 \\
$\begin{array}{l}\text { Incontinencia } \\
\text { esfuerzo }\end{array}$ & 2 & 4 & 3 \\
Inestabilidad vesical & 6 & 4 & 2 \\
\hline
\end{tabular}

\section{DISCUSIÓN}

El Síndrome Uretral (incontinencia, urgencia, disuria) afecta a pacientes del sexo femenino, ocasionando disconfort en la vida diaria de estas pacientes. Muchas de ellas han recibido múltiples tratamientos (psiquiátricos, médicos, instrumentales, y cirugias) y no mejoran, siendo un problema para muchos urólogos que vemos este tipo de pacientes. El síndrome uretral afecta al $7-12 \%$ de las mujeres mayores de 65 años. Dos tercios de estas pacientes tienen inestabilidad vesical idiopática ${ }^{1}$.

Las pacientes que presentan este tipo de Síndrome Uretral suelen tener una media de 55 años (35-81 años), edad en las que se ve afectación cerebro-vascular y problemas de circulación donde las antagonistas del calcio pueden tener su lugar.

Los antagonistas del calcio inhiben el paso del calcio al músculo liso vascular, lo cual ayuda a eliminar las molestias de urgencia. Son lípidosolubles, y se excretan por la bilis. Los antagonistas del calcio, en especial la Flunarizina, reducen la incontinencia de orina y la urgencia. Palmer $^{1}$ informo que no cambia la frecuencia de las micciones, aunque en esta serie se observó que las espacía. Palmer et al., en su serie de 14 pacientes que acudieron con incontinencia de orina, frecuencia urinaria e inestabilidad del detrusor, las trataron con $20 \mathrm{mg}$ de Flunarizina, una dosis diaria, observando que la capacidad vesical se ve incrementada, así como la primera sensación en la cistometria se ve retardada.

Se han utilizado otras drogas tales como el Flavoxato, y la Nifedipina, que son también calcio antagonistas, los cuales incrementan la capacidad vesical ${ }^{2}$.

Como toda droga, los antagonistas del calcio no dejan de tener sus efectos colaterales en el tratamiento prolongado, tales como vejiga hipotónica, somnolencia, incremento de la sudoración, edema periférico, somnolencia, cefalea, dolor abdominal, náuseas, sequedad de boca, insomnio, rash cutáneo, aumento de peso.

Entre las contraindicaciones de los antagonistas del calcio se ha informado hipersensibilidad a la flunarizina, insuficiencia hepática, o renal severa, cardiopatías descompasadas, depresión severa, enfermedad de Parkinson, embarazo y lactancia. No debe de administrarse con alcohol porque puede potencializar los efectos sedantes de los depresores del SNC.

\section{REFERENCIAS}

1. Palmer JH, Worth PHL, Exton-Smith AN.: Flunarizine: A once-daily therapy for urinary incontinence. Lancet. 1981 Aug 8;2(8241):279-281.

2. Rud T, Andersson KE, Ulmsten U.: Effects of nifedipine in women with unstable bladders. Urol Int. 1979;34(6):421-429.

Dr. E.A. Granados Loarca

(Trabajo recibido el 30 de marzo 2005) 